\title{
Predator-Prey Models on Interaction between Computer Worms, Trojan Horse and Antivirus Software Inside a Computer System
}

\author{
Munna Kumar ${ }^{1}$, Bimal Kumar Mishra ${ }^{2}$ and T. C. Panda ${ }^{3}$ \\ ${ }^{1}$ Research Scholar,Department of Mathematics, Utkal University, \\ Bhubaneswar, Orissa, India - 751004 \\ ${ }^{2}$ Department of Applied Mathematics, Birla Institute of Technology \\ Mesra, Ranchi, India - 835215 \\ ${ }^{3}$ Former Principal, Orissa Engineering College, Bhubaneswar \\ Orissa, India - 752050 \\ 1'indian68mk@gmail.com, 2drbimalmishra@gmail.com, \\ ${ }^{3}$ tc_panda@yahoo.com
}

\begin{abstract}
In this paper an attempt has been made to develop Predator-prey Models on interaction between Computer worms, Trojan horse and antivirus software inside a computer system. Five mathematical models are proposed to study the Predator-prey system inside a computer system. In mathematical model 1, the prey consists of uninfected files, whereas, the predator consists of computer worms. In mathematical model 2, the infected files constitute the prey and anti-virus software is the predator. In mathematical model 3, Effect of new or updated antivirus software on such computer worms which are quarantine or not completely recovered by the lower version of installed antivirus software in the system is studied which switches the rate of infection to zero. In mathematical model 4, Reactivation of computer worms when they are in the latent class is mathematically formulated. In mathematical model 5, we have attempt to develop and understand the recent attack of the Trojan horse Backdoor. Kihomchi and Trojan.Zbot!gen74 and its removal by newly available tool NPE.exe. The stability of the result is stated in terms of the Jacobian of the system and the basic reproduction number is also well defined for all models. Numerical methods and MATLAB are employed to solve and simulate the system of equations developed and analysis of the models gives remarkable exposure.
\end{abstract}

Keywords: Predator-prey model; Computer worms; Trojan horse; Basic reproduction number; Antivirus software; Quarantine; Latency time; Self-replication

\section{Introduction}

The computer network is epidemic in nature due to attack of malicious objects like worms, viruses and Trojans horses. The attacking behaviours of different malicious codes are different. The development of communication network have made computer more and more important in our daily life. The computers malicious codes are small program develop to damage computer system, erasing data, stealing information or modifying normal operations. Their action throughout a network can be studied by using classical epidemiological models for diseases propagation.

A virus, worm or Trojan horse can (like HIV) be latent, only to become active after a certain period. This is called a 'logic bomb'. These three classes of computer malware can also have hundreds of variants or several slightly modified versions, with parallel microbial diversity $[1,2]$. 
A computer worm is similar to a computer virus in many aspects, except that it is a self-contained program "that is able to spread functional copies of itself or its segments to other computer systems" without depending on another program to host its code. The basic objective of the worm is to gain access to another machine so that it can replicate itself on the new machine and reproduce further. To avoid the attack of computer worms, we need some strong software and we know that antivirus software is a commonly used method for controlling computer worms. The transmission of worms in computer network is epidemic in nature and is analogous to biological epidemic diseases.

A trojan horse is a program that contains or installs a malicious program. Trojan horses may appear to be interesting programs (or at the very least harmless) to an unsuspecting user, but are actually harmful when executed. Unlike viruses, Trojan horses do not replicate themselves but they can be just as destructive. One of the most insidious types of Trojan horse is a program that claims to rid the computer of viruses but instead introduces viruses into the computer.

The malicious code (machine language program) which has the ability to spread through various sources may spread in any one or all of the following ways:

- $\quad$ The spreading medium may be a malicious attachment to an email.

- Malware medium may constitute a USB pen drive, a floppy disk, a CD or any secondary media which is commonly used by almost all computer professionals.

Whenever a vulnerable node in the network is attacked, some of the malware (malicious agent) have the property of self-replicating within the same node by a factor known as replication factor. It denotes the number of malicious objects a single malicious object generates over a fixed period of time. Virus and worms do have this characteristic.

An acute epidemic occurs due to infectious malcode designed to actively spread from host to host over a network. When the user executes an infected program, the worms may take control of the computer and infect additional files. After the worms completed its mischief, it would transfer control to the host program and allow it to function normally.

Conventional antivirus systems are knowledge-based, so if the system doesn't recognize a piece of code as malware, it won't block it [3].

This malicious code when tries to enter into a protected (secured system) system installed with an Intrusion Detection System (IDS), it analyzes the unknown binary code whether it is malicious or not. An IDS, enabled with signature analysis and an ad-on security alarm is deployed to monitor the network and host system activities [4, 5]. IDS's are supported by a knowledge-based evaluation system to focus on real threatening alerts and assist in post attack forensics. The job done by such knowledge-based systems is to filter out false positives and rank the severity of attacks. The Knowledge based stores all well-known exploits and system vulnerability information together with the corresponding security solutions. It tunes the IDS with the known signatures and sends the proper action to the Artificial Immune System (AIS). This AIS attempts to classify network traffic as either self (normal file or uninfected file) or non-self (malicious or infected file) and provide a proactive protection via negative selection [6].With great insight into the virus signature, the immune system disinfects the infected files verifying the occurrence of the attack, or otherwise it issues an isolated alert and quarantines the infected data into its blind spots. Therefore, by correlating these alerts, the quarantined data is kept under a latency period. During this period the antivirus update is incorporated and finally, the data kept under latency is recovered to its original normal form.

The various epidemic models on the transmission of malicious objects in computer network as per the spreading behaviors and nature of the malicious objects has developed by Mishra et al., [1, 2,7]. Predicting computer worms and Trojan horse outbreaks is extremely difficult due to human nature of the attacks but more importantly, detecting outbreaks early with a low probability of false alarms seems quiet difficult. It is possible to characterize essential properties of the attacks by developing various epidemic models [7]. 
The subsequent materials are organized in this pattern: Section 1 deals with introduction of the paper. Section 2 formulates the model. Section 2.1 Formulation of Model 1: Initial phase of an Infection. Section 2.2 Formulation of Model II: Secondary Phase of Infection (Effect of antivirus software). Section 2.3 Formulation of Model III: Effect of new antivirus software on such worms which are quarantine. Section $\mathbf{2 . 4}$ Formulation of Model IV: Reactivation of computer worms after they are in latent class. Finally, Section 2.5 Formulation of Model V: Recent Attack by Trojan horse Backdoor.Kihomchi and Trojan.Zbot!gen74 and its Mathematical approach. Section 3 Discussion and Conclusion.

\section{Nomenclature:}

W: number of Worms / Trojan horse in the computer system.

$S$ : number of uninfected target files.

I: number of infected files.

c: Replicating factor.

b: Birth of uninfected files by users.

$\delta$ : Death rate of a worm / Trojan horse.

$\mu$ : Natural Death rate of an uninfected file.

$\alpha$ : Death rate of infected files.

$\beta$ : Infectious contact rate, i.e., the rate of infection per susceptible and per infective.

$\mathrm{R}_{0}$ : Basic reproductive ratio.

$\mathrm{R}_{0}^{\prime}$ : Basic reproductive ratio in the presence of antivirus software.

A: Response of antivirus software, which immunes the system.

$\rho$ : Rate at which antivirus software is run, which is constant.

$\eta$ : Death rate of antivirus software.

$\gamma:$ Rate at which antivirus software cleans the infected files.

$k:$ Immune response parameter.

$\mathrm{I}_{1}$ : productive infected class.

$\mathrm{I}_{2}$ : latent infected class.

$\theta$ : Per capita rate at which files at latent infected class leave for productive infected class.

$\lambda_{1}$ : Probability of entering productive infected class.

$\lambda_{2}$ : Probability of entering latent infected class.

U: Un-infectious Trojan horse which start to be produced from the infected files I.

\section{The following assumptions are made to characterize the model:}

- Worm is replicated by the infected files.

- Worms / Trojan horse die at a specific rate $\delta$. Death of a worm / Trojan horse equivalently mean to say the complete recovery of infected files from worms / Trojan horse when antivirus software is run in the computer node for a specific session.

- The uninfected files are constantly being produced or developed by the users at a rate $b$.

- Uninfected files die at a constant rate $\mu$ (natural death). Death of a file equivalently mean to say that the file become irrelevant (garbage) after a certain interval of time.

- Infected files die at a specific rate $\mu+\alpha$, where $\mu$ is natural death rate and $\alpha$ the death rate of the file (files get damaged and unable to be recovered after the run of antivirus software) due to infection from the worm.

- Death of antivirus software equivalently mean to say the present version of the software is incapable of identifying the attack of new worms / Trojan horse. 


\section{Formulation of the Model}

Worms / Trojan horse is a computer program that operates on behalf of a potential intruder to aid in attacking a system or network which behave like infectious diseases and are epidemic in nature. Some of the worms have the characteristic of self-replicating and some of them get enter in the latent class and reactivate after certain duration. When a system gets attacked by the worms and Trojan horse, antivirus software is run to immune the system. During this process some of the infected files get fully recovered, whereas, some of them are quarantined, may be due to the lower version of the antivirus software installed. Then for this situation a higher version or new antivirus software is run to get a full recovery. We try to develop Mathematical models for these situations [8].

\subsection{Formulation of Model 1: Initial phase of an Infection}

Worms can be in any form; like attachment of malicious executable file, malicious hyperlink and phishing by clicking incidentally or wrongly an attachment of malicious executable file can infect the system and hijack various files (command files, executable files, kernel.dll, etc.) in the node for its own replication. It then leaves a specific file and the process is repeated. Worms behave like infectious disease and are epidemic in nature. They target different file types of the attacked computer for this purpose.

In the computer system, to derive the model equation, there are three classes: $\mathrm{W}$ be the number of worms (predators), $S$ be the number of uninfected files (prey) [8] and I be the number of infected files. The relationship between the computer Worms and uninfected file is analogous to the relationship between predator and prey as given in the classical work of Lotka-Volterra $[9,10]$.

On the basis of the assumptions, we get the following system of equations.

$$
\begin{aligned}
& \frac{d W}{d t}=c I-\delta W \\
& \frac{d S}{d t}=b-\mu S-\beta S W \\
& \frac{d I}{d t}=\beta S W-(\mu+\alpha) I
\end{aligned}
$$

Where $b$ is the birth rate of uninfected files by users, $\delta$ is the death rate of worms, $\mu$ is the natural death rate of uninfected files, $\alpha$ is the death rate of infected files. Worms give rise to infected files at a rate $\beta S$ and each infected file gives rise to a worm (selfreplication) at a rate $c$. Then,

$\{$ Rate of change of $S\}=\{$ net rate of growth of $S$ without predation $\}-\{$ rate of loss due of $\mathrm{S}$ to predation $\}$ and

$\{$ Rate of change of $\mathrm{W}\}=\{$ net rate of growth of $\mathrm{W}$ due to predation $\}-\{$ net rate of loss of W without prey

2.1.1. Basic Reproduction Number $\left(\mathbf{R}_{\mathbf{0}}\right)$ : Let, $\mathrm{R}_{0}$ be the basic reproductive ratio for the computer worms; defined to be the expected number of worms that one worm gives rise to an uninfected file population.

Since the model has two infected classes (W, I). So to get $\mathrm{R}_{0}$, we take only two equations from the system (1) corresponding to these classes. That is,

$$
\frac{d W}{d t}=c I-\delta W
$$


$\frac{d I}{d t}=\beta S W-(\mu+\alpha) I$

On linearization, we get, $\left[\begin{array}{c}\frac{d W}{d t} \\ \frac{d I}{d t}\end{array}\right]=(\mathrm{F}-\mathrm{V})\left[\begin{array}{l}W \\ I\end{array}\right]$, where, $\mathrm{F}$, a matrix of rates of infection and $\mathrm{V}$, a matrix of rates of transmission, are defined by, $\mathrm{F}=\left[\begin{array}{ll}0 & 0 \\ \beta & 0\end{array}\right]$ and

$$
\mathrm{V}=\left[\begin{array}{cc}
\delta & -c \\
0 & \mu+\alpha
\end{array}\right]
$$

Then the basic reproductive number $R_{0}$ is defined as the dominant eigen value of $\mathrm{F} \mathrm{V}^{-1}$.

That is, $\quad R_{0}=\frac{\beta c}{\delta(\mu+\alpha)}$

The criterion for the spread of the computer worms is $R_{0}>1$.

2.1.2. Solutions and Stability: The system (1) is defined on the closed, positive invariant set $\mathrm{D}=\{(\mathrm{W}, \mathrm{S}, \mathrm{I}) ; \mathrm{W}, \mathrm{S}, \mathrm{I} \geq 0: W+S+I=1\}$ which has two possible equilibriums, first, the worms free equilibrium, $\mathrm{D}_{0}=(0,1,0)$ and second, the endemic equilibrium $D^{*}=\left(W^{*}, S^{*}, I^{*}\right)$ which is the interior of $\mathrm{D}$ and can be obtained by taking all the equations of system (1) equal to zero. That is,

$$
W^{*}=\frac{\beta b c-\delta \mu(\mu+\alpha)}{\delta \beta(\mu+\alpha)}, S^{*}=\frac{\delta(\mu+\alpha)}{\beta c}=\frac{1}{R_{0}}, \text { and } I^{*}=\frac{\beta b c-\delta \mu(\mu+\alpha)}{\beta c(\mu+\alpha)}
$$

For $R_{0}>1$, The susceptible population $\mathrm{S}$ (uninfected files) is reduced by the attack until each worm is expected to give rise to exactly one new worm, $R_{0} S^{*}=1$.

This we assume as the Initial phase of an infection.

Theorem 1: The system (1) is locally asymptotically stable if all its eigen values are negative.

Proof: By using system (1), the Jacobian can be taken as,

$$
J=\left[\begin{array}{ccc}
-\delta & 0 & c \\
0 & -\mu & 0 \\
0 & 0 & -(\mu+\alpha)
\end{array}\right]
$$

Solving, we get the eigen values, $-\delta,-\mu,-(\mu+\alpha)$ which all are negative. So, the system (1) is locally asymptotically stable.

2.1.3 Numerical Methods and Simulation: We present the numerical result using Runge-Kutta method of order 4 and MATLAB are employed to solve and simulate the 
system (1) and the behavior of the different classes with respect to time are observed which is depicted in figures 1 and we observe that the system is asymptotically stable. The network is assumed to have initial values: $\mathrm{W}=3 ; \mathrm{S}=45 ; \mathrm{I}=2$.

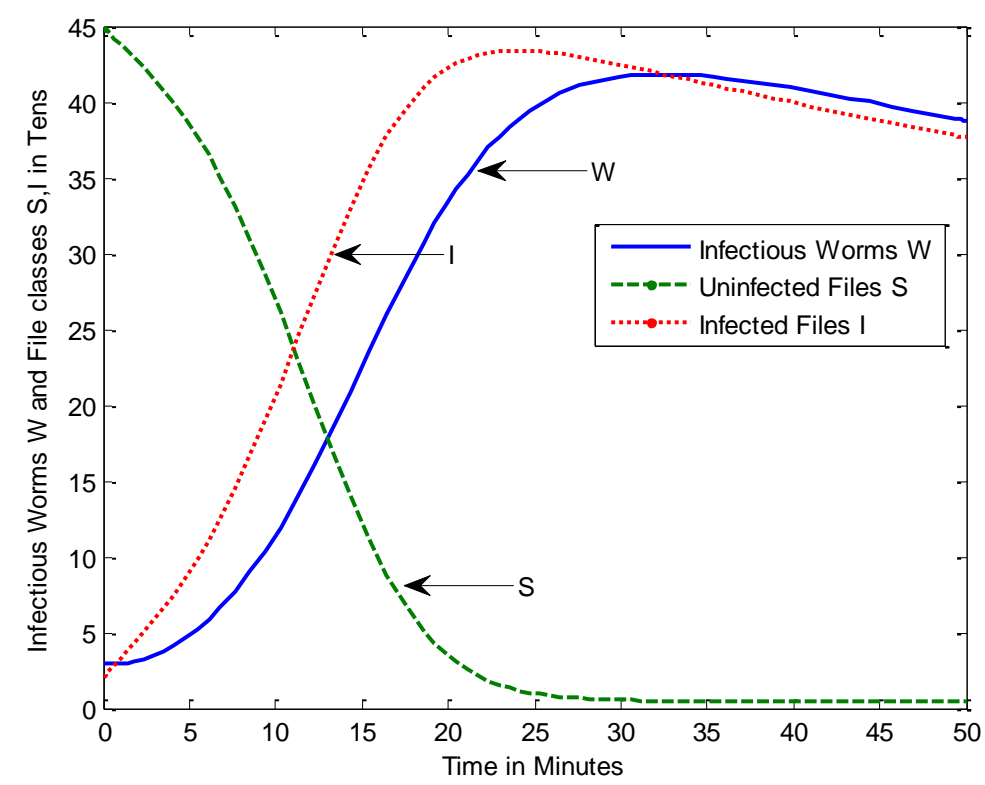

Figure 1. Dynamical Behavior of the System (1) for Different Classes when $c=0.2 ; \delta=0.2 ; b=0.2 ; \mu=0.001 ; \beta=0.01 ; \alpha=0.01$;

Figure 1 shows the uninfected files (prey) are reduced by the attack of infectious worms (predators). Infectious worms (predators) are going to increase over a period of time and then getting loss due to lack of uninfected files (prey) i.e. natural death. Infected files are increasing due to uninfected files being infected and after sometimes decreased due to death of infected files.

\subsection{Formulation of Model II: Secondary Phase of Infection (Effect of antivirus software)}

An interaction between worms and antivirus software inside a system can be modeled by considering the response in a computer system due to antivirus software A.

In the computer system, to derive the model equation, there are four classes: $\mathrm{W}$ be the number of worms, $\mathrm{S}$ be the number of uninfected files, I be the number of infected files (prey) and A be the antivirus software (predators). We take linear functional response of A to I.

On the basis of the assumptions, we get the following system of equations.

$$
\begin{aligned}
& \frac{d W}{d t}=c I-\delta W \\
& \frac{d S}{d t}=b-\mu S-\beta S W \\
& \frac{d I}{d t}=\beta S W-(\mu+\alpha) I-\gamma I A
\end{aligned}
$$


$\frac{d A}{d t}=\rho-\eta A$

Where $b$ is the birth rate of uninfected files by users, $\delta$ is the death rate of Worms, $\mu$ is the natural death rate of uninfected files, $\alpha$ is the death rate of infected files, $\rho$ is a constant rate at which antivirus software runs, $\eta$ is the death rate of antivirus software(which mean to say that the antivirus software is incapable to identify the attack of new worms), $\gamma$ is a rate at which antivirus software cleans the infected files, Worms give rise to infected files at a rate $\beta S$ and each infected file gives rise to a worm (selfreplication) at a rate $c$.

\subsubsection{Basic Reproduction Number $\left(\mathbf{R}^{\prime}{ }_{0}\right)$}

Let, $\mathrm{R}_{0}^{\prime}$ be the basic reproductive ratio in the presence of the antivirus software.

Since the model has two infected classes (W, I). So to get $\mathrm{R}_{0}$, we take only two equations from the system (3) corresponding to these classes. That is,

$$
\begin{aligned}
& \frac{d W}{d t}=c I-\delta W \\
& \frac{d I}{d t}=\beta S W-(\mu+\alpha) I-\gamma I A
\end{aligned}
$$

On linearization, we get, $\left[\begin{array}{c}\frac{d W}{d t} \\ \frac{d I}{d t}\end{array}\right]=(\mathrm{F}-\mathrm{V})\left[\begin{array}{l}W \\ I\end{array}\right]$, where, $\mathrm{F}$, a matrix of rates of infection and $\mathrm{V}$, a matrix of rates of transmission, are defined by, $\mathrm{F}=\left[\begin{array}{ll}0 & 0 \\ \beta & 0\end{array}\right]$ and $\mathrm{V}=\left[\begin{array}{cc}\delta & -c \\ 0 & \mu+\alpha+\gamma\end{array}\right]$

Then the basic reproductive number $\mathrm{R}_{0}^{\prime}$ is defined as the dominant eigen value of $\mathrm{F} \mathrm{V}^{-1}$.

That is, $\quad \mathrm{R}_{0}^{\prime}=\frac{\beta c}{\delta(\mu+\alpha+\gamma)}$

Then we observe that the infection persists as long as $\mathrm{R}_{0}^{\prime}>1$.

In order for the immune response to clear the infection we need the immune response parameter $\kappa$ to satisfy $\kappa>\frac{\mu+\alpha}{\mu}\left(R_{0}-1\right)$

where $\kappa=\frac{\gamma \rho}{\mu \eta}$

2.2.2 Solutions and Stability: The system (3) is defined on the closed, positive invariant set $\mathrm{D}=\{(\mathrm{W}, \mathrm{S}, \mathrm{I}, \mathrm{A}) ; \mathrm{W}, \mathrm{S}, \mathrm{I}, \mathrm{A} \geq 0: W+S+I+A=1\}$ which has two possible equilibriums, first, the worms free equilibrium, $\mathrm{D}_{0}=(0,1,0,1)$ and second, the endemic 
equilibrium $D^{*}=\left(W^{*}, S^{*}, I^{*}, A^{*}\right)$ which is the interior of $\mathrm{D}$ and can be obtained by taking all the equations of system (3) equal to zero. That is,

$$
\begin{array}{ll}
W^{*}=\frac{\beta b c \eta-\delta \mu^{2} \eta-\delta \mu \alpha \eta-\gamma \delta \rho \mu}{\beta \delta(\mu \eta+\alpha \eta+\gamma \rho)} & , \\
I^{*}=\frac{\beta b c \eta-\delta \mu^{2} \eta-\delta \mu \alpha \eta-\gamma \delta \rho \mu}{\beta c(\mu \eta+\alpha \eta+\gamma \rho)} & \text { and } A^{*}=\frac{\rho}{\eta}
\end{array}
$$

Theorem 1: The system (3) is locally asymptotically stable if all its eigen values are negative.

Proof: By using system (3), the Jacobian can be taken as,

$$
J=\left[\begin{array}{cccc}
-\delta & 0 & c & 0 \\
0 & -\mu & 0 & 0 \\
0 & 0 & -(\mu+\alpha+\gamma A) & 0 \\
0 & 0 & 0 & -\eta
\end{array}\right]
$$

Solving, we get the eigen values, $-\delta,-\mu,-(\mu+\alpha+\gamma A),-\eta$, which all are negative. So, the system (3) is locally asymptotically stable.

\subsubsection{Numerical Methods and Simulation}

We present the numerical result using Runge-Kutta method of order 4 and MATLAB are employed to solve and simulate the system (3) and the behavior of the different classes with respect to time are observed which is depicted in figures 2 and we observe that the system is asymptotically stable. The network is assumed to have initial values: $\mathrm{W}=25 ; \mathrm{S}=5 ; \mathrm{I}=30: \mathrm{A}=0$.

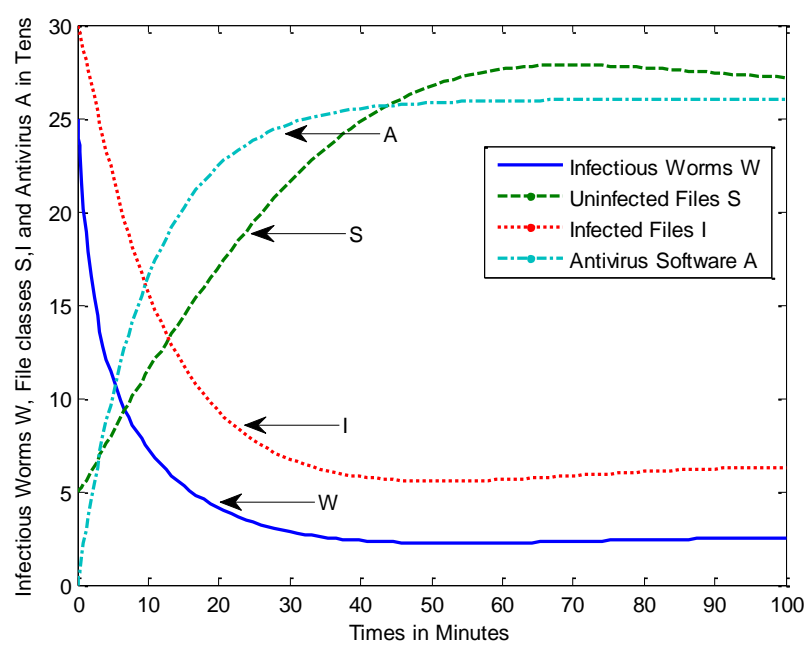

Figure 2. Dynamical Behavior of the System (3) for Different Classes when $c=0.2 ; \delta=0.5 ; \quad b=2.5 ; \mu=0.055 ; \beta=0.015 ; \alpha=0.055 ; \gamma=0.002 ; \rho=2.6 ; \eta=0.1$;

Figure 2 shows the uninfected files are increasing as infected files (prey) are cleaning by antivirus software (predators). Infectious worms are also getting loss due to run of antivirus software. Infected files (prey) are decreasing since antivirus software (predators) 
cleans it. Antivirus software (predators) is running but being to death due to incapable to identify the attack of new worms.

\subsection{Formulation of Model III: Effect of New Antivirus Aoftware on such Worms which are Quarantine}

We assume a case where the installed present version of the antivirus software on the computer node is incapable of identifying the attack of new worms and not completely cleaned which send to quarantine from the infected files. An available $100 \%$ efficient and updated version of antivirus software has to be run for the complete recovery of infected files from worms. This updated version of antivirus software switches $\beta$ to zero. Therefore the equations for the subsequent dynamics of the infected files and free worms from equation (1) is expressed as

$$
\begin{aligned}
& \frac{d W}{d t}=c I-\delta W \\
& \frac{d S}{d t}=b-\mu S \\
& \frac{d I}{d t}=-(\mu+\alpha) I
\end{aligned}
$$

(6)

Where $b$ is the birth rate of uninfected files by users, $\delta$ is the death rate of worms, $\mu$ is the natural death rate of uninfected files, $\alpha$ is the death rate of infected files. Each infected file gives rise to a worm (self-replication) at a rate $c$.

\subsubsection{Basic Reproduction Number $\left(\mathbf{R}_{0}\right)$}

Since the model has two infected classes (W, I). So to get $\mathrm{R}_{0}$, we take only two equations from the system (6) corresponding to these classes. That is,

$$
\begin{aligned}
& \frac{d W}{d t}=c I-\delta W \\
& \frac{d I}{d t}=-(\mu+\alpha) I
\end{aligned}
$$

On linearization, we get, $\left[\begin{array}{c}\frac{d W}{d t} \\ \frac{d I}{d t}\end{array}\right]=(\mathrm{F}-\mathrm{V})\left[\begin{array}{l}W \\ I\end{array}\right]$, where, $\mathrm{F}$, a matrix of rates of infection and $\mathrm{V}$, a matrix of rates of transmission, are defined by, $\mathrm{F}=\left[\begin{array}{ll}0 & 0 \\ 0 & 0\end{array}\right]$ and $\mathrm{V}=\left[\begin{array}{cc}\delta & -c \\ 0 & \mu+\alpha\end{array}\right]$

Then the basic reproductive number $R_{0}$ is defined as the dominant eigen value of $\mathrm{F} \mathrm{V}^{-1}$. The criterion for the spread of the computer worms is $R_{0}>1$. 
2.3.2 Solutions and Stability: The system (6) is defined on the closed, positive invariant set $\mathrm{D}=\{(\mathrm{W}, \mathrm{S}, \mathrm{I}) ; \mathrm{W}, \mathrm{S}, \mathrm{I} \geq 0: W+S+I=1\}$ which has two possible equilibriums, first, the worms free equilibrium, $\mathrm{D}_{0}=(0,1,0)$ and second, the endemic equilibrium $D^{*}=\left(W^{*}, S^{*}, I^{*}\right)$ which is the interior of $\mathrm{D}$ and can be obtained by taking all the equations of system (6) equal to zero.

That is, $W^{*}=0, S^{*}=\frac{b}{\mu}$ and $I^{*}=0$

Theorem 1: The system (6) is locally asymptotically stable if all its eigen values are negative.

Proof: By using system (6), the Jacobian can be taken as,

$$
J=\left[\begin{array}{ccc}
-\delta & 0 & c \\
0 & -\mu & 0 \\
0 & 0 & -(\mu+\alpha)
\end{array}\right]
$$

Solving, we get the eigen values, $-\delta,-\mu,-(\mu+\alpha)$ which all are negative. So, the system (6) is locally asymptotically stable.

We further assume that the half-life of the worms is much less than that of the worms producing files. Then,

$$
\begin{aligned}
& I=I_{0} e^{-(\mu+\alpha) t} \\
& W=\frac{W_{0}\left(\delta e^{-(\mu+\alpha) t}-(\mu+\alpha) e^{-\delta t}\right)}{\delta-(\mu+\alpha)}
\end{aligned}
$$

From Equation (7) we are able to say that the number of infected files falls exponentially. The behavior of $\mathrm{W}$ follows from the assumption on half-lives, so that $\mu+\alpha$ $<<\delta$, that is, the amount of free worms falls exponentially after a shoulder phase.

2.3.3 Numerical Methods and Simulation: We present the numerical result using Runge-Kutta method of order 4 and MATLAB are employed to solve and simulate the system (6) and the behavior of the different classes with respect to time are observed which is depicted in figures 3 and we observe that the system is asymptotically stable. The network is assumed to have initial values: $\mathrm{W}=5 ; \mathrm{S}=10 ; \mathrm{I}=7$. 


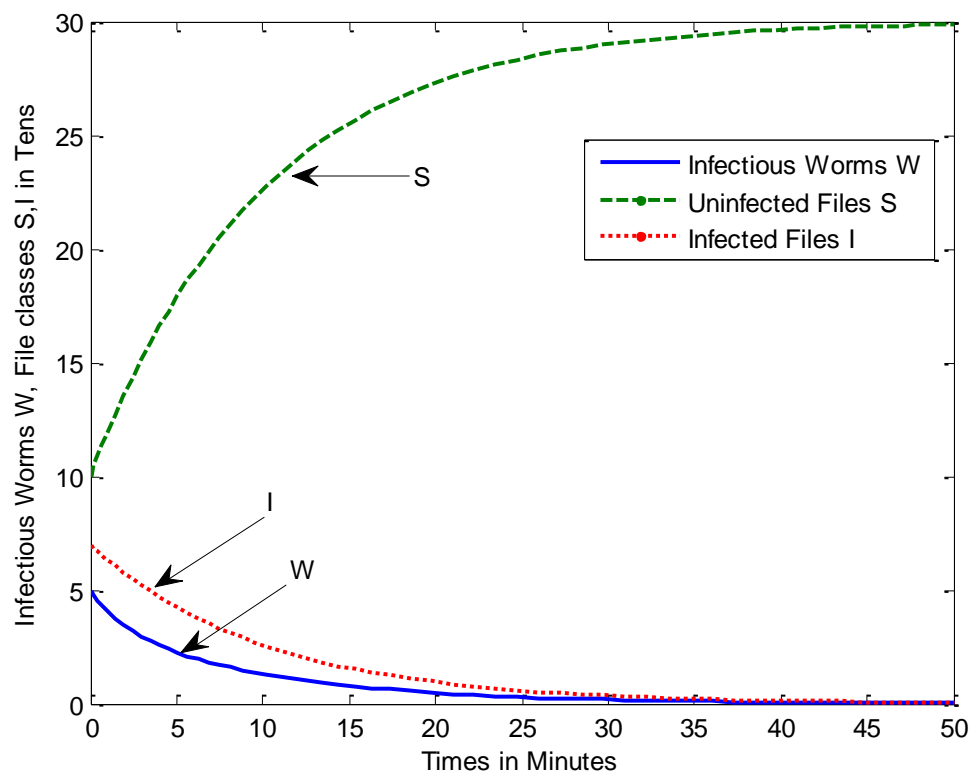

Figure 3. Dynamical Behavior of the System (6) for Different Classes when $c=0.2 ; \delta=0.5 ; b=3.0 ; \mu=0.1 ; \alpha=0.0001$;

Figure 3 shows the uninfected files are increasing as infected files (prey) are completely recovered from worms by updated antivirus software (predators). Infectious worms are also getting loss to zero due to run of updated antivirus software. Infected files (prey) decreased to zero since updated antivirus software (predators) cleans it.

\subsection{Formulation of Model IV: Reactivation of Computer Worms after they are in Latent Class}

Some worms enter a latent class on their infection when they attack the computer node. They do not produce new worms in this latent class, but may later be reactivated to do so. Only the files in the productive infected class $\mathrm{I}_{1}$ produce worms, and files at latent infected class $I_{2}$ leave for $I_{1}$ at a per capita rate $\theta$. Thus we get the following system of equations:

$$
\begin{aligned}
& \frac{d W}{d t}=c I-\delta W \\
& \frac{d S}{d t}=b-\mu S-\beta S W \\
& \frac{d I_{1}}{d t}=\psi_{1} \beta S W-\left(\mu_{1}+\alpha_{1}\right) I_{1}+\theta I_{2} \\
& \frac{d I_{2}}{d t}=\psi_{2} \beta S W-\left(\mu_{2}+\alpha_{2}\right) I_{2}-\theta I_{2}
\end{aligned}
$$

Where $\mathrm{b}$ is the birth rate of uninfected files by users, $\delta$ is the death rate of worms, $\mu$ is the natural death rate of uninfected files, $\Psi_{1}, \mu_{1}, \alpha_{1}$ are the probability of entering ,the natural death rate and the death rate of productive infected files, $\Psi_{2}, \mu_{2}, \alpha_{2}$ are the probability of entering, the natural death rate and the death rate of latent infected files, $\theta$ is Per capita rate at which files at latent infected class leave for productive infected class, Worms give rise to infected files at a rate $\beta S$ and each infected file gives rise to a worm 
(self-replication) at a rate $c$. Infected files at class $I_{2}$ produce worms in class $I_{1}$ at a rate $\theta$ for a time $\frac{1}{\theta+\left(\mu_{2}+\alpha_{2}\right)}$.

\subsubsection{Basic Reproduction Number $\left(\mathbf{R}_{\mathbf{0}}\right)$}

Since the model has three infected classes $\left(\mathrm{W}, \mathrm{I}_{1}, \mathrm{I}_{2}\right)$. So to get $\mathrm{R}_{0}$, we take only three equations from the system (8) corresponding to these classes. That is,

$$
\begin{aligned}
& \frac{d W}{d t}=c I-\delta W \\
& \frac{d I_{1}}{d t}=\psi_{1} \beta S W-\left(\mu_{1}+\alpha_{1}\right) I_{1}+\theta I_{2} \\
& \frac{d I_{2}}{d t}=\psi_{2} \beta S W-\left(\mu_{2}+\alpha_{2}\right) I_{2}-\theta I_{2}
\end{aligned}
$$

On linearization, we get, $\left[\begin{array}{c}\frac{d W}{d t} \\ \frac{d I_{1}}{d t} \\ \frac{d I_{2}}{d t}\end{array}\right]=(\mathrm{F}-\mathrm{V})\left[\begin{array}{c}W \\ I_{1} \\ I_{2}\end{array}\right]$, where, $\mathrm{F}$, a matrix of rates of infection and $\mathrm{V}$, a matrix of rates of transmission, are defined by, $\mathrm{F}=\left[\begin{array}{ccc}0 & 0 & 0 \\ \beta & 0 & 0 \\ \beta & 0 & 0\end{array}\right]$ and $\mathrm{V}=\left[\begin{array}{ccc}\delta & -c & 0 \\ 0 & \mu_{1}+\alpha_{1} & -\theta \\ 0 & 0 & \mu_{2}+\alpha_{2}+\theta\end{array}\right]$

Then the basic reproductive number $R_{0}$ is calculated by the formula $\left|F V^{-1}-\lambda I\right|=0$

That is, $\quad R_{0}=\frac{\beta c\left(\mu_{2}+\alpha_{2}+2 \theta\right)}{\delta\left(\mu_{1}+\alpha_{1}\right)\left(\mu_{2}+\alpha_{2}+\theta\right)}$.

2.4.2 Solutions and Stability: The system (8) is defined on the closed, positive invariant set $\mathrm{D}=\left\{\left(\mathrm{W}, \mathrm{S}, \mathrm{I}_{1}, \mathrm{I}_{2}\right) ; \mathrm{W}, \mathrm{S}, \mathrm{I}_{1}, \mathrm{I}_{2} \geq 0: W+S+\mathrm{I}_{1}+\mathrm{I}_{2}=1\right\}$ which has two possible equilibriums, first, the worms free equilibrium, $\mathrm{D}_{0}=(0,1,0,0)$ and second, the endemic equilibrium $D^{*}=\left(W^{*}, S^{*}, I_{1}^{*}, I_{2}^{*}\right)$ which is the interior of $\mathrm{D}$ and can be obtained by taking all the equations of system (8) equal to zero. That is,

$$
W^{*}=\frac{\beta b c\left[\psi_{2} \theta+\psi_{1}\left(\mu_{2}+\alpha_{2}+\theta\right)\right]-\mu_{1} \mu \delta\left(\mu_{2}+\alpha_{2}+\theta\right)-\alpha_{1} \mu \delta\left(\mu_{2}+\alpha_{2}+\theta\right)}{\beta \delta\left(\mu_{1}+\alpha_{1}\right)\left(\mu_{2}+\alpha_{2}+\theta\right)},
$$




$$
\begin{aligned}
& S^{*}=\frac{\delta\left(\mu_{1}+\alpha_{1}\right)\left(\mu_{2}+\alpha_{2}+\theta\right)}{\beta c\left[\psi_{2} \theta+\psi_{1}\left(\mu_{2}+\alpha_{2}+\theta\right)\right]}, \\
& I_{1}^{*}=\frac{\beta b c\left[\psi_{2} \theta+\psi_{1}\left(\mu_{2}+\alpha_{2}+\theta\right)\right]-\mu_{1} \mu \delta\left(\mu_{2}+\alpha_{2}+\theta\right)-\alpha_{1} \mu \delta\left(\mu_{2}+\alpha_{2}+\theta\right)}{\beta c\left(\mu_{1}+\alpha_{1}\right)\left(\mu_{2}+\alpha_{2}+\theta\right)} \text { and } \\
& I_{2}{ }^{*}=\frac{\psi_{2}\left[\beta b c\left[\psi_{2} \theta+\psi_{1}\left(\mu_{2}+\alpha_{2}+\theta\right)\right]-\mu_{1} \mu \delta\left(\mu_{2}+\alpha_{2}+\theta\right)-\alpha_{1} \mu \delta\left(\mu_{2}+\alpha_{2}+\theta\right)\right]}{\beta c\left[\psi_{2} \theta+\psi_{1}\left(\mu_{2}+\alpha_{2}+\theta\right)\right]\left(\mu_{2}+\alpha_{2}+\theta\right)}
\end{aligned}
$$

Theorem 1: The system (8) is locally asymptotically stable if all its eigen values are negative.

Proof: By using system (8), the Jacobian can be taken as,

$$
J=\left[\begin{array}{cccc}
-\delta & 0 & c & 0 \\
0 & -\mu & 0 & 0 \\
0 & 0 & -\left(\mu_{1}+\alpha_{1}\right) & \theta \\
0 & 0 & 0 & -\left(\mu_{2}+\alpha_{2}+\theta\right)
\end{array}\right]
$$

Solving, we get the eigen values, $-\delta,-\mu,-\left(\mu_{1}+\alpha_{1}\right),-\left(\mu_{2}+\alpha_{2}+\theta\right)$ which all are negative. So, the system (8) is locally asymptotically stable.

2.4.3 Numerical Methods and Simulation: We present the numerical result using Runge-Kutta method of order 4 and MATLAB are employed to solve and simulate the system (8) and the behavior of the different classes with respect to time are observed which is depicted in figures 4 and we observe that the system is asymptotically stable. The network is assumed to have initial values: $\mathrm{W}=0 ; \mathrm{S}=45 ; \mathrm{I}_{1}=0 ; \mathrm{I}_{2}=2$.

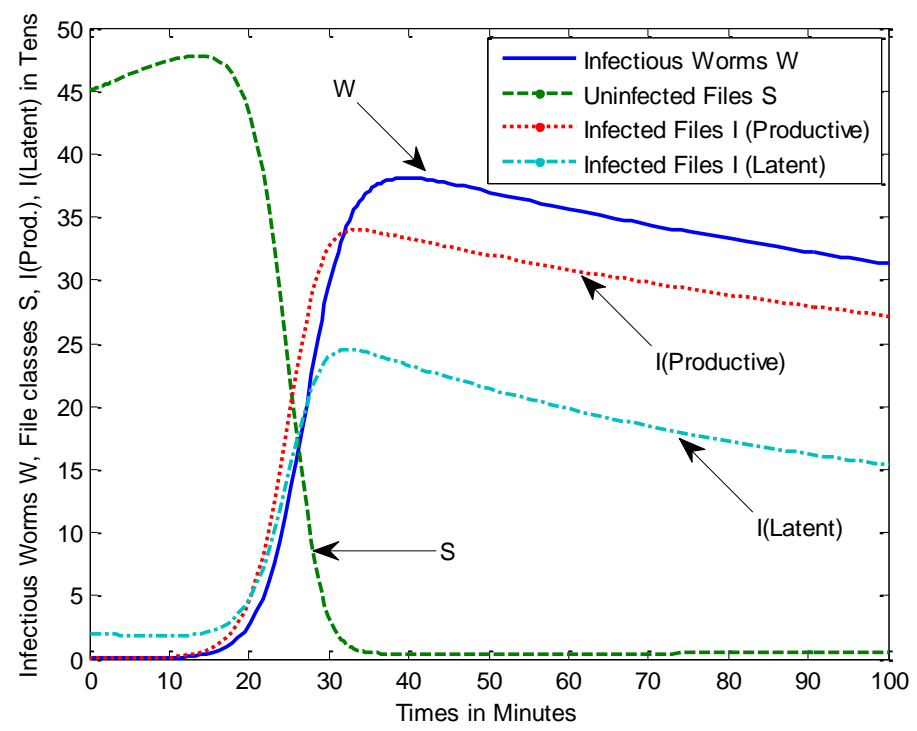

Figure 4: Dynamical Behavior of the System (8) for Different Classes when $c=0.4 ; \delta=0.35 ; b=0.3 ; \mu=0.001 ; \beta=0.02 ; \Psi_{1}=0.7 ; \quad \Psi_{2}=0.5 ; \mu_{1}=0.01 ;$ $\mu_{2}=0.006 ; \quad \alpha_{1}=0.001 ; \quad \alpha_{2}=0.008 ; \theta=0.001 ;$

Figure 4 shows the Infectious worms in latent class are increasing after reactivating with productive infected files. Uninfected files are decreasing as productive infected files 
produce worms. Productive Infected files are increasing and latent infected files leave for productive infected files.

\subsection{Formulation of Model V: Recent Attack by Trojan Horse Backdoor.Kihomchi and Trojan.Zbot!gen74 and its Mathematical Approach}

On April 15, 2014 Backdoor.Kihomchi and Trojan.Zbot!gen74 malicious objects of type Trojan Horse having infection length of 327,680 bytes affected Windows 95, Windows 98, Windows 2000, Windows Me, Windows NT, Windows XP, Windows 7, Windows Vista, Windows Server 2003, Windows Server 2008.

Backdoor.Kihomchi is a Trojan horse program that opens a backdoor on the compromised computer, downloads a remote file and executes it. It logs key strokes and send stolen key strokes to the remote location.

Trojan.Zbot!gen74 is also a Trojan horse program that opens a backdoor and attempts to steal confidential information from the compromised computer. It specifically targets system sensitive information, online credentials, and banking details, but can be customized through the toolkit to gather any sort of information. It may also download configuration files and updates from the Internet. These can later be updated to target other information, if the attacker so wishes.

The tool NPE.exe is known as Norton Power Eraser and it is developed by Symantec Corporation is designed to remove the infections of Backdoor.Kihomchi [11] and Trojan.Zbot!gen74 [12].

NPE.exe tool meant to remove the deadly Backdoor.Kihomchi and Trojan.Zbot!gen74 prevent infected files from producing infectious Trojan horse.

We assume that $U$ are the un- infectious Trojan horse which start to be produced from the infected files I after the tool NPE.exe is run. Infectious Trojan horse are still present, and die as before, but are no longer produced.

Under this assumption we can be modeled the system as:

$$
\begin{aligned}
& \frac{d W}{d t}=-\delta W \\
& \frac{d S}{d t}=b-\mu S \\
& \frac{d I}{d t}=-(\mu+\alpha) I \\
& \frac{d U}{d t}=c I-\delta U
\end{aligned}
$$

Where $b$ is the birth rate of uninfected files by users, $\delta$ is the death rate of Trojan horse, $\mu$ is the natural death rate of uninfected files, $\alpha$ is the death rate of infected files. Each infected file gives rise to a worm (self-replication) at a rate $c$.

\subsubsection{Basic Reproduction Number $\left(\mathbf{R}_{\mathbf{0}}\right)$}

Since the model has two infected classes (W, I). So to get $\mathrm{R}_{0}$, we take only two equations from the system (10) corresponding to these classes. That is,

$$
\frac{d W}{d t}=-\delta W
$$


$\frac{d I}{d t}=-(\mu+\alpha) I$

On linearization, we get, $\left[\begin{array}{c}\frac{d W}{d t} \\ \frac{d I}{d t}\end{array}\right]=(\mathrm{F}-\mathrm{V})\left[\begin{array}{c}W \\ I\end{array}\right]$, where, F, a matrix of rates of infection and $\mathrm{V}$, a matrix of rates of transmission, are defined by, $\mathrm{F}=\left[\begin{array}{ll}0 & 0 \\ 0 & 0\end{array}\right]$ and $\mathrm{V}=\left[\begin{array}{cc}\delta & 0 \\ 0 & \mu+\alpha\end{array}\right]$

Then the basic reproductive number $R_{0}$ is defined as the dominant eigen value of $\mathrm{F} \mathrm{V}^{-1}$.

2.5.2 Solutions and Stability: The system (10) is defined on the closed, positive invariant set $\mathrm{D}=\{(\mathrm{W}, \mathrm{S}, \mathrm{I}, \mathrm{U}) ; \mathrm{W}, \mathrm{S}, \mathrm{I}, \mathrm{U} \geq 0: W+S+I+U=1\}$ which has two possible equilibriums, first, the Trojan horse free equilibrium, $\mathrm{D}_{0}=(0,1,0,0)$ and second, the endemic equilibrium $D^{*}=\left(W^{*}, S^{*}, I^{*}, U^{*}\right)$ which is the interior of $\mathrm{D}$ and can be obtained by taking all the equations of system (10) equal to zero. That is, $W^{*}=0$, $S^{*}=\frac{b}{\mu}, I^{*}=0$ and $U^{*}=0$.

Theorem 1: The system (10) is locally asymptotically stable if all its eigen values are negative.

Proof: By using system (10), the Jacobian can be taken as,

$J=\left[\begin{array}{cccc}-\delta & 0 & 0 & 0 \\ 0 & -\mu & 0 & 0 \\ 0 & 0 & -(\mu+\alpha) & 0 \\ 0 & 0 & c & -\delta\end{array}\right]$

Solving, we get the eigen values, $-\delta,-\mu,-(\mu+\alpha),-\delta$, which all are negative. So, the system (10) is locally asymptotically stable.

We assume that the uninfected file population $\mathrm{S}$ remains roughly constant for a given time-scale, that is, $S=S^{*}=\frac{\delta(\mu+\alpha)}{c \beta}$ and that $(\mu+\alpha) \ll \delta$ System (10) becomes a linear system which is integrated to have

$$
\begin{aligned}
& W=W_{0} e^{-\delta t} \\
& I=I_{0} \frac{\left.(\mu+\alpha) e^{-\delta t}-\delta e^{-(\mu+\alpha) t}\right)}{(\mu+\alpha)-\delta} \text { when }(\mu+\alpha)<<\delta
\end{aligned}
$$




$$
U=U_{0} \frac{\delta}{\delta-(\mu+\alpha)}\left(\frac{\delta}{\delta-(\mu+\alpha)}\left(e^{-(\mu+\alpha) t}-e^{-\delta t}\right)-(\mu+\alpha) e^{-\delta t}\right)
$$

From (11) it is clear that the total amount $W+U$ of free Trojan horse falls exponentially after a shoulder phase.

2.5.3 Numerical Methods and Simulation: We present the numerical result using Runge-Kutta method of order 4 and MATLAB are employed to solve and simulate the system (10) and the behavior of the different classes with respect to time are observed which is depicted in figures 5 and we observe that the system is asymptotically stable. The network is assumed to have initial values: $\mathrm{W}=45 ; \mathrm{S}=5 ; \mathrm{I}=30 ; \mathrm{U}=30$.

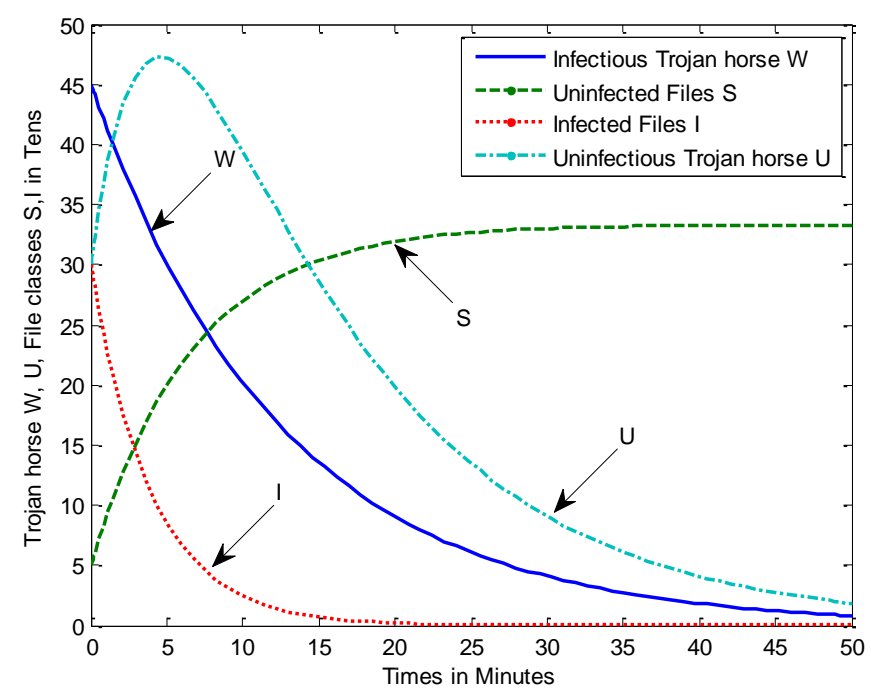

Figure 5: Dynamical Behavior of the System (10) for Different Classes when $c=0.4 ; \delta=0.08 ; \quad b=5.0 ; \mu=0.15 ; \alpha=0.1 ;$

Figure 5 shows the Infectious Trojan horse are still present and decreasing to no longer produce after the tool NPE.exe is run. Uninfected files are increasing due to lack of infectious Trojan horse and then remains roughly constant for a given time-scale. Infected files are decreasing and Un-infectious Trojan horse start to be produced.

\section{Discussion and Conclusion}

A predator- prey mathematical model of different phases of infection of files and their immune system have been formulated. We have assumed that the worms / Trojan horse possess a non-negligible latent period $\&$ infected files will stay in the latent period before they become infectious. Reproduction number $\left(\mathrm{R}_{\mathrm{o}}\right)$ and $\left(\mathrm{R}_{0}^{\prime}\right)$ in presence of immune system, equilibria and their stability are also found. Verification and validation of our model parameter has been analyzed by simulating the system. In Model I by the help of basic reproduction number obtained in (2) for Initial phase of infection discusses the criterion for the spread of the computer worms, that is, $R_{0}>1$. The susceptible population $\mathrm{S}$ (uninfected files) is reduced by the attack until each worm is expected to give rise to exactly one new worms, $R_{0} S^{*}=1$. In Model II the basic reproductive ratio in the presence of the antivirus software is defined by (4) and in order for the immune response to clear the infection we need the immune response parameter $\kappa$ to satisfy $\kappa>$ $\frac{\mu+\alpha}{\mu}\left(R_{0}-1\right)$. In Model III for the worms which are quarantined by the installed 
antivirus software, we assume that updated antivirus software is available and is $100 \%$ efficient. When this updated antivirus software is run, from equation (7) we are able to say that the number of infected files falls exponentially. The behavior of $\mathrm{W}$ follows from the assumption on half-lives, so that $\mu+\alpha<<\delta$, that is, the amount of free worms falls exponentially after a shoulder phase. In Model IV discussion is also made for those worms which enter a latent class on their infection and in this class they do not produce new worms, but may later be reactivated to do so. Infected files at class $I_{2}$ produce worms in class $I_{1}$ at a rate $\theta$ for a time $\frac{1}{\theta+\left(\mu_{2}+\alpha_{2}\right)}$ and the reproductive ratio is also obtained. In Model $\mathbf{V}$ the tool NPE.exe is run to prevent infected files from producing infectious Trojan horse. It is clear that the total amount $\mathrm{W}+\mathrm{U}$ of free Trojan horse falls exponentially after a shoulder phase from equation (11). The study will be helpful to the software organization in developing efficient antivirus software.

\section{References}

[1] B. Kumar Mishra, D. Saini, "Mathematical models on computer viruses", Applied

[2] Mathematics and Computation, 187 (2007), pp. 929-936.

[3] B. Kumar Mishra , N. Jha, "Fixed period of temporary immunity after run of anti-malicious objects software on computer nodes", Applied Mathematics and Computation, 190 (2007), pp. 1207-1212.

[4] Masud, Mohammad M., Khan, Latifur and Thuraisingham, Bhavani, "A Knowledge-based Approach to detect new Malicious Executables". In the proceedings of the Second Secure Knowledge Management Workshop (SKM) (2006), Brooklyn, NY, USA.

[5] A,K Jones, and R.S Sielken, "Computer System Intrusion detection: a survey", Technical report, Computer Science Department, University of Virginia,(2000).

[6] Yu, J., Reddy, R., Selliah, S., Reddy, S., Bharadwaj, V. and Kankanahalli S.,"TRINETR: An Architecture for Collaborative Intrusion Detection and Knowledge-Based Alert Evaluation",In Advanced Engineering Informatics Journal, Special Issue on Collaborative Environments for Design and Manufacturing. Editor: Weiming Shen. Volume 19, Issue 2, April(2005), Elsevier Science, pp. 93-101.

[7] J. Yu, Y.V. Ramana Reddy, Sentil Selliah,Srinivas Kankanahalli, Sumitra Reddy and Vijayanand Bhardwaj, "A Collaborative Architecture for Intrusion Detection Systems with Intelligent Agents and Knowledge based alert Evaluation", In the Proceedings of IEEE $8^{\text {th }}$ International Conference on Computer Supported Cooperative work in Design, (2004), 2: ,pp. 271- 276.

[8] B. Kumar Mishra, D.K Saini, "SEIRS epidemic model with delay for transmission of malicious objects in computer network", Applied Mathematics and Computation, 188 (2007), pp. 1476-1482.

[9] N F. Britton, "Essential Mathematical Biology", Springer-Verlag, London, (2003).

[10] A. J Lotka, , "Elements of Physical Biology", Williams and Wilkins, Baltimore, (1925), Reissued as "Elements of Mathematical Biology", Dover, New York, (1956).

[11] V. Volterra, , "Variazioni e fluttazioni del numero d'individui in specie animali conviventi", Mem. Acad. Sci. Lincei, (1926), 2:3, pp. 1-13.

[12] http://www.symantec.com/security_response/writeup.jsp?docid=2014-041515-2137-99.

[13] http:// www.symantec.com/security_response/writeup.jsp?docid=2014-041711-2459-99,

\section{Authors}

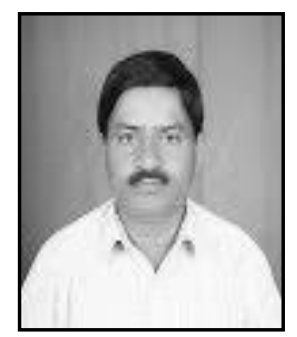

Munna Kumar is a research scholar pursuing his $\mathrm{Ph}$. D. degree. He received his Master's in Mathematics from Vinoba Bhave University, Hazaribag, India. His research interests include Mathematical Modeling on cyber attack and defense. 


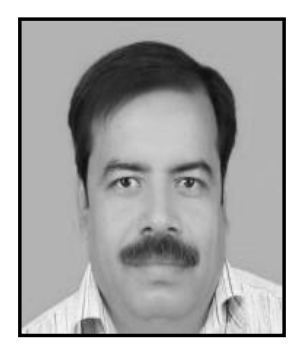

Bimal Kumar Mishra is a Professor in the Department of Mathematics, Birla Institute of Technology, Mesra, Ranchi, India. He received his Master's in Mathematics and Master's in Operational Research from University of Delhi, India and earned his Ph. D. from Vinoba Bhave University, Hazaribag, India in 1997. He was awarded D. Sc. In 2007 from Berhampur University, India. He has published more than 100 research papers in journals of high repute and conference proceedings. His research interests include Nonlinear Analysis and Bifurcation and presently working in the area of Cyberattack and defence.

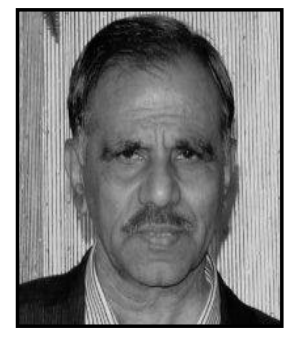

T. C. Panda is a Retired Professor in the Department of Mathematics, Berhampur University, India. He is the President of Orissa Mathematical Society, Bhubaneshwar Area, India. He has published in journals of high repute and supervised several $\mathrm{Ph}$. D. scholars. His research areas include Fluid Mechanics, and Nonlinear Analysis. 